WellBeing International

WBI Studies Repository

$1-2020$

\title{
Social learning in solitary juvenile sharks
}

\author{
Catarina Vila Pouca \\ Macquarie University \\ Dennis Heinrich \\ Flinders University \\ Charlie Huveneers \\ Flinders University \\ Culum Brown \\ Macquarie University
}

Follow this and additional works at: https://www.wellbeingintlstudiesrepository.org/socbeh

Part of the Animal Studies Commons, Behavior and Ethology Commons, and the Comparative Psychology Commons

\section{Recommended Citation}

Pouca, C. V., Heinrich, D., Huveneers, C., \& Brown, C. (2020). Social learning in solitary juvenile sharks.

Animal Behaviour, 159, 21-27. DOI: https://doi.org/10.1016/j.anbehav.2019.10.017

This material is brought to you for free and open access by WellBeing International. It has been accepted for inclusion by an authorized administrator of the WBI Studies Repository. For more information, please contact wbisr-info@wellbeingintl.org.

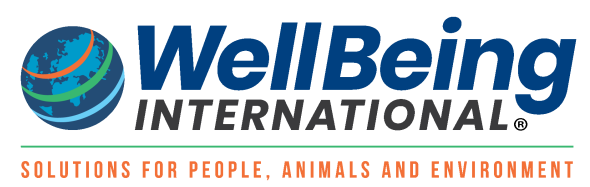




\title{
Social learning in solitary juvenile sharks
}

\author{
Catarina Vila Pouca ${ }^{\text {a, }}$, Dennis Heinrich ${ }^{\text {b }}$, Charlie Huveneers ${ }^{\text {b }}$, Culum Brown ${ }^{\text {a }}$ \\ a Department of Biological Sciences, Macquarie University, Sydney, Australia \\ ${ }^{\mathrm{b}}$ College of Science and Engineering, Flinders University, Bedford Park, South Australia, Australia
}

\section{A R T I C L E I N F O}

\section{Article history:}

Received 5 July 2019

Initial acceptance 2 September 2019

Final acceptance 27 October 2019

MS. number: 19-00456

\section{Keywords:}

cognition

conformity

elasmobranchs

gregarious

sociality

\begin{abstract}
Social learning can be a shortcut for acquiring locally adaptive information. Animals that live in social groups have better access to social information, but gregarious and nonsocial species are also frequently exposed to social cues. Thus, social learning might simply reflect an animal's general ability to learn rather than an adaptation to social living. Here, we investigated social learning and the effect of frequency of social exposure in nonsocial, juvenile Port Jackson sharks, Heterodontus portusjacksoni. We compared (1) Individual Learners, (2) Sham-Observers, paired with a naïve shark, and (3) Observers, paired with a trained demonstrator, in a novel foraging task. We found that more Observers learnt the foraging route compared to Individual Learners or Sham-Observers, and that Individual Learners took more days to learn. Training frequency did not affect learning rate, suggesting acquisition occurred mostly between training bouts. When demonstrators were absent, $30 \%$ of observers maintained their performance above the learning criterion, indicating they retained the acquired information. These results indicate that social living is not a prerequisite for social learning in elasmobranchs and suggest social learning is ubiquitous in vertebrates.
\end{abstract}

() 2019 The Association for the Study of Animal Behaviour. Published by Elsevier Ltd. All rights reserved.
Animals often share similar needs and challenges with other individuals, such as finding food, the best habitat or suitable mates, or avoiding predators. The ability to learn from the observation or interaction with another animal, termed social learning, can therefore be adaptive as it can save individuals the costs of learning by trial-and-error (Galef \& Laland, 2005; Laland, 2004). Social learning was thought to be limited by species' sociality levels since social groups provide better access to social information (Giraldeau \& Lefebvre, 1996). However, even solitary or gregarious animals are often exposed to other individuals (e.g. neighbouring conspecifics, competitors, mates) and social cues might be just one among many cues available in the environment that lead to learning (Heyes, 2012; Webster \& Laland, 2017). Support for this hypothesis has come from a few species, including noncolonial insects, nonsocial reptiles and nonschooling fish (Coolen, Dangles, \& Casas, 2005; Kis, Huber, \& Wilkinson, 2015; Noble, Byrne, \& Whiting, 2014; Webster \& Laland, 2017). For example, social and nonschooling species of fish showed no differences in social information use and social learning in a foraging task (Webster \& Laland, 2017). These results imply that social learning is not an adaptation for social living and

\footnotetext{
* Correspondence: C. Vila Pouca, Department of Biological Sciences, Macquarie University, 209 Culloden Road, Marsfield, Sydney, NSW 2122, Australia.

E-mail address: catarinavilapouca@gmail.com (C. Vila Pouca).
}

question whether asocial and social learning have separate evolutionary origins.

The relative efficacy of social learning is likely to be influenced by certain characteristics of demonstrators and observers, as well as by the effectiveness of the demonstration which influences the opportunity for naïve individuals to learn. Group size alone can enhance skill acquisition in a social context (e.g. Brown \& Warburton, 1999), but a high proportion of knowledgeable demonstrators in the group and scrounging effects have been shown to hamper learning (Brown \& Laland, 2002; Lefebvre \& Giraldeau, 1994; Vilhunen, Hirvonen, \& Laakkonen, 2005). In addition, the quality of the demonstration also plays a role in the spread of information through the group; for example, observer guppies, Poecilia reticulata, paired with well-trained demonstrators performed worse than those paired with poorly trained demonstrators, probably because the well-trained demonstrators were too fast to be followed (Swaney, Kendal, Capon, Brown, \& Laland, 2001). Collectively, these results suggest a complex link between the extent of social exposure and observer learning performance that warrants further investigation.

Elasmobranchs are a key taxon to investigate the perceptive and cognitive mechanisms of social learning as well as its evolutionary origins among vertebrates since they are one of the oldest vertebrate groups and have very diverse life history traits (Naylor, Ryburn, Fedrigo, \& Lopez, 2005; Schluessel, 2015). Yet to date, 
social learning has only been tested in one shark and one ray species (Negaprion brevirostris, Guttridge et al., 2013; Potamotrygon falkneri, Thonhauser et al., 2013). While many species of sharks are not traditionally viewed as social animals, recent evidence suggests that they can have sophisticated social lives (Jacoby, Croft, \& Sims, 2012; Mourier, Vercelloni, \& Planes, 2012). There is, however, considerable variation in social structure between species and often between life history stages; for example, adult Port Jackson sharks, Heterodontus portusjacksoni, rest in socially structured groups, while juveniles do not associate with conspecifics (Mourier, Bass, Guttridge, Day, \& Brown, 2017a; Powter \& Gladstone, 2009; Vila Pouca \& Brown, 2019).

In this study, we tested whether juvenile Port Jackson sharks use social information from conspecifics and assessed the effect of frequency of social exposure in learning performance. One group of naïve focal sharks was trained individually. Two additional groups could observe, and interact with, either sharks that were trained to gain access to food through one of two arbitrary routes or naïve sharks with no previous experience in the task. We predicted that sharks paired with knowledgeable demonstrators would learn faster than individual learners and sharks exposed to sham demonstrators, and that higher frequency of social demonstration would result in faster learning.

\section{METHODS}

\section{Subjects and Apparatus}

Sixty Port Jackson shark eggs were collected from Jervis Bay, New South Wales, and hatched in captivity. Juvenile sharks were housed in groups of two to four at the Sydney Institute of Marine Science (SIMS), Australia, in 1000-litre sea water tanks at ambient temperature for at least 6 months prior to the experiment. Tanks had continuous circulation of sea water, aeration and a thin layer of sand on the bottom. PVC structures and fake kelp provided shelter and enrichment. Sea water was pumped directly from Sydney harbour at ambient temperature (ranging $18.2-22.4{ }^{\circ} \mathrm{C}$, April to June 2016 and 2018). Prior to the experiment, sharks were fed small pieces of squid, fish and prawns ad libitum three times per week. The experimental tank was adjacent to the housing tanks and the room had a natural light/dark cycle.

The experimental tank $(180 \times 100 \mathrm{~cm}$ and $40 \mathrm{~cm}$ high $)$ was divided lengthways by a black partition (Fig. 1a). The sharks could access the other side of the tank through two holes $(22 \times 10 \mathrm{~cm})$ in the partition, which were visually marked to facilitate discrimination (Fig. 1b). At one end of the tank ('Task side', Fig. 1a), a sliding mesh wall was used to create a starting compartment. At the opposite end of the tank ('Reward side', Fig. 1a), a black divider separated the reward locations. Sharks were rewarded using long aquarium tongs and the daily food intake per individual during the experimental period was equivalent to $2 \%$ of their wet body weight in squid, Loligo opalescens, pieces. Water inflow was provided from both sides of the tank, with multiple small inflow points along the bottom of the tank, and water outflow was located on the reward end of the tank (Fig. 1a).

\section{Experimental Pairs}

Focal sharks were pseudorandomly assigned to one of three treatments: Individual Learners $(N=12)$, Sham-Observers $(N=14$; paired with naïve Sham-Demonstrators) and Observers $(N=20$; paired with trained Demonstrators). We used 14 naïve ShamDemonstrators (one per Sham-Observer) to ensure the exposure of Sham-Demonstrators to the task matched that of their naïve Sham-Observer counterpart. The Demonstrators $(N=8)$ were selected from the Individual Learners' group and reused two to four times with different focal Observers. Sham-Observer/ShamDemonstrator and Observer/Demonstrator pairs were chosen according to housing tank, ensuring that sharks in each pair were housed in separate tanks and had no opportunity to become familiar with each other. Half of the sharks were trained to use the route on the right side of the partition and half trained to use the route on the left. In addition, sharks were trained in one of two schedules: low frequency ( 3 trials/day; ' $3 T$ ') or high frequency (6 trials/day; '6T'), following a balanced design.

\section{Procedure}

Sharks were moved from the housing tank to the experimental arena each day. Before the start of the experiment, each shark was pre-exposed to the experimental tank for 3 consecutive days to allow them to overcome any stress associated with moving tanks and swimming through the holes in the partition, as familiarity with the test environment facilitates learning (Brown, 2001). Familiarization sessions lasted $45 \mathrm{~min}$, and in the last 15 min sharks were fed in random locations in the experimental tank to become familiar with receiving food from aquarium tongs. Sharks from the paired treatments (Sham-Observer/Sham-Demonstrator and Observer/Demonstrator) were acclimated in pairs to become familiar with sharing the experimental tank with another individual.

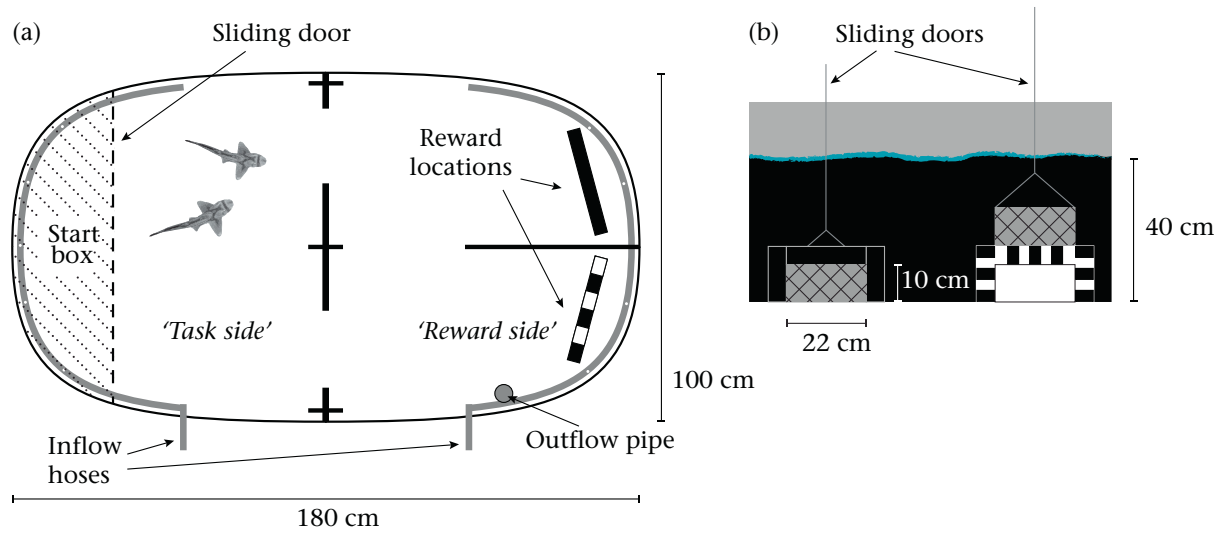

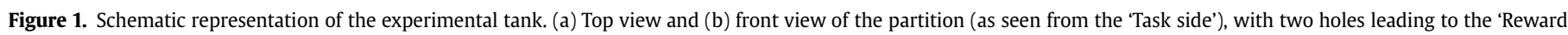
side'. 
Training sessions began with 5 min of acclimation in the 'Task side' of the arena (both routes were blocked with sliding doors). Trials began in the start box. After 30-60 s, the box was opened. Sham-Demonstrators/Demonstrators could swim multiple times between the Task and Reward sides during a trial, but only the first correct choice was rewarded. Focal sharks could only choose one route per trial. The trial ended when the focal shark swam through the correct route and was rewarded, swam through the incorrect route, or did not swim through the partition within $90 \mathrm{~s}$. At the end of each training session, sharks were given a random interval of 3-6 min to settle and fed the remainder of the food allocated for the day inside the start box.

Individual Learners were trained for another experiment (Heinrich, Vila Pouca, Brown, \& Huveneers, 2019) for 21 days, of which the first 10 were pretraining (incorrect route blocked with a sliding door). Since we expected social learning to occur faster, the incorrect route was never blocked for Sham-Observers/Observers and training lasted 10 days. On days 11-12, Sham-Observers/ Observers were tested in isolation.

Sharks were considered successful in the task if they made 10/12 correct choices and had fewer than $50 \%$ null trials over the training stage. To be able to compare Individual Learners and the paired treatments, we considered that Individual Learners failed the task if they did not reach criterion within 12 days, which was the maximum number of days the paired treatments ran the task. However, to compare the number of days taken to reach criterion, we included all Individual Learners that reached criterion within the 21 days of the experiment.

Trials were video recorded and scored using BORISv.2.62 (Friard \& Gamba, 2016). We recorded whether the focal shark chose the correct or incorrect route and the latency to choose. Additionally, we recorded Sham-Demonstrator/Demonstrator's first choice and latency, and total correct and incorrect crossings per trial.

\section{Statistical Analyses}

Statistical analyses were performed in R v.3.5.2 (R Core Team, 2016). We compared Demonstrators and Sham-Demonstrators in: (1) choice of first crossing (correct $=1$; incorrect $=0$ ) using a generalized linear mixed-effect model (GLMM; binomial distribution); (2) latency of first choice using a linear mixed-effect model (LMM; Gaussian); (3) total partition crossings per trial (GLMM, Poisson); and (4) proportion of correct crossings per trial (GLMM, binomial). All models included Training Day as an additional predictor variable and Individual ID as a random effect.

For Individual Learners, Sham-Observers, and Observers, we compared: (5) number of sharks that learnt the task (learnt $=1$; failed $=0$ ) using a generalized linear model (GLM, binomial distribution); (6) number of days until learning criterion (GLM, Poisson); (7) choice of route (correct $=1$; incorrect $=0$; GLMM, binomial); (8) latency of choice (LMM, Gaussian); (9) latency of correct choice (LMM, Gaussian); (10) choice of route during the test days (GLMM, binomial); and (11) motivation (null trials over total trials per individual; GLM, binomial). Individual Learners were not included in (7-10) since days 1-10 were pretraining and cannot be compared to regular training days. All models included Training Frequency ('3T', '6T') as an additional predictor variable; models (7-10) further included Training Day and Individual ID, and models (9-10) an interaction between Learning Treatment and Training Day. See the Appendix for further details.

\section{Ethical Note}

Egg collection occurred under NSW Fisheries permit P08/00104.2. The experiments were approved by the Macquarie University
Animal Ethics Committee (ARA 2014-003). Sharks were kept under optimal conditions and remained healthy during the experiments. Tanks were scrubbed clean at least once a week. During nonexperimentation periods, sharks were fed on a mixed diet of squid, fish and prawns ad libitum three times per week, and given vitamin supplements (Elasmo tabs $150 \mathrm{mg}$, Vetafarm) every 2 weeks. All sharks were released at their original site of capture a few days after the experiment.

\section{RESULTS}

\section{Sham-Demonstrators and Demonstrators}

Demonstrator sharks chose the correct route on the first crossing more often, were faster in making a choice, made multiple crossings through the partition more often and had a higher proportion of correct crossings over all crossings per trial compared to Sham-Demonstrators (Fig. 2, Appendix Table A1), indicating that they were more effective in demonstrating the task.

\section{Individual Learners, Sham-Observers and Observers}

Fifteen Observer sharks (75\%) met the learning criterion compared to three Sham-Observer (21\%) and one Individual Learner (8\%; Fig. 3a). There was no effect of Training Frequency (Table 1, Appendix Table A2). An additional eight Individual Learners reached criterion after day 12 and were included in the remaining analyses. Individual Learners took significantly more days to reach criterion compared to Observers and Sham-Observers (Fig. 3b), and sharks running 3 trials/day (3T) or 6 trials/day (6T) took the same number of days to reach criterion (Table 1, Appendix Table A3). Observer sharks chose the correct route more often than Sham-Observers and, even though Sham-Observers were faster in crossing the partition on the initial days of the task, Observers got faster over time (Fig. 3c-d, Table 1).

When tested without demonstration on days $11-12,30 \%(6 / 20)$ of Observers and 21\% (3/14) of Sham-Observers performed above criterion, with no differences between the groups in choice of route (Table 1). Of the 14 Observer sharks that failed without demonstration, six did not make a choice in more than half of the trials while eight participated in most trials.

Individual Learners seemed to have higher motivation to participate in the task compared to the paired treatments, but no significant effect of Learning Treatment, Training Frequency or an interaction was detected, probably because of the very high individual variation within groups (Appendix Table A1, Fig. A1).

\section{DISCUSSION}

We have shown that nonsocial, juvenile Port Jackson sharks use social information and socially learn a new foraging route. Observers paired with trained Demonstrators learnt faster than Individual Learners or Sham-Observers (paired with a naïve conspecific). The frequency of training had no impact on learning rates.

Given that juvenile Port Jackson sharks are solitary animals, our results indicate that social living is not a prerequisite for social learning in elasmobranchs and support the hypothesis that social learning might simply reflect an animal's general ability to learn. Together with a few studies documenting social learning in nongrouping species (Coolen et al., 2005; Kis et al., 2015; Noble et al., 2014; Webster \& Laland, 2017), these results suggest that social learning is likely to be ubiquitous across vertebrates. Even among solitary or gregarious species, individuals occasionally encounter each other and might provisionally aggregate when exploiting 

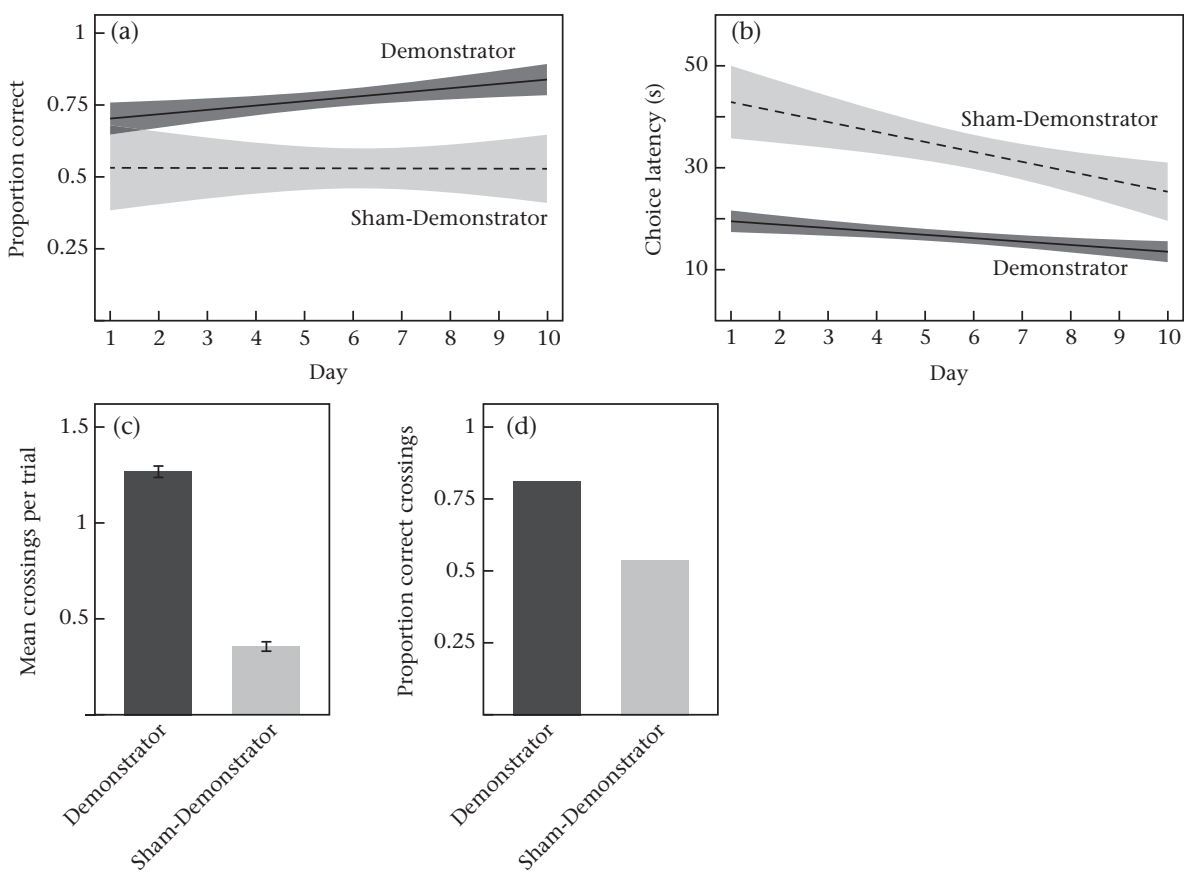

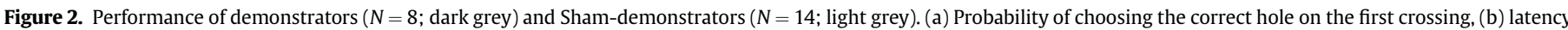
to cross the partition for the first time, (c) mean number of crossings per trial and (d) proportion of correct crossings over all crossings per trial.
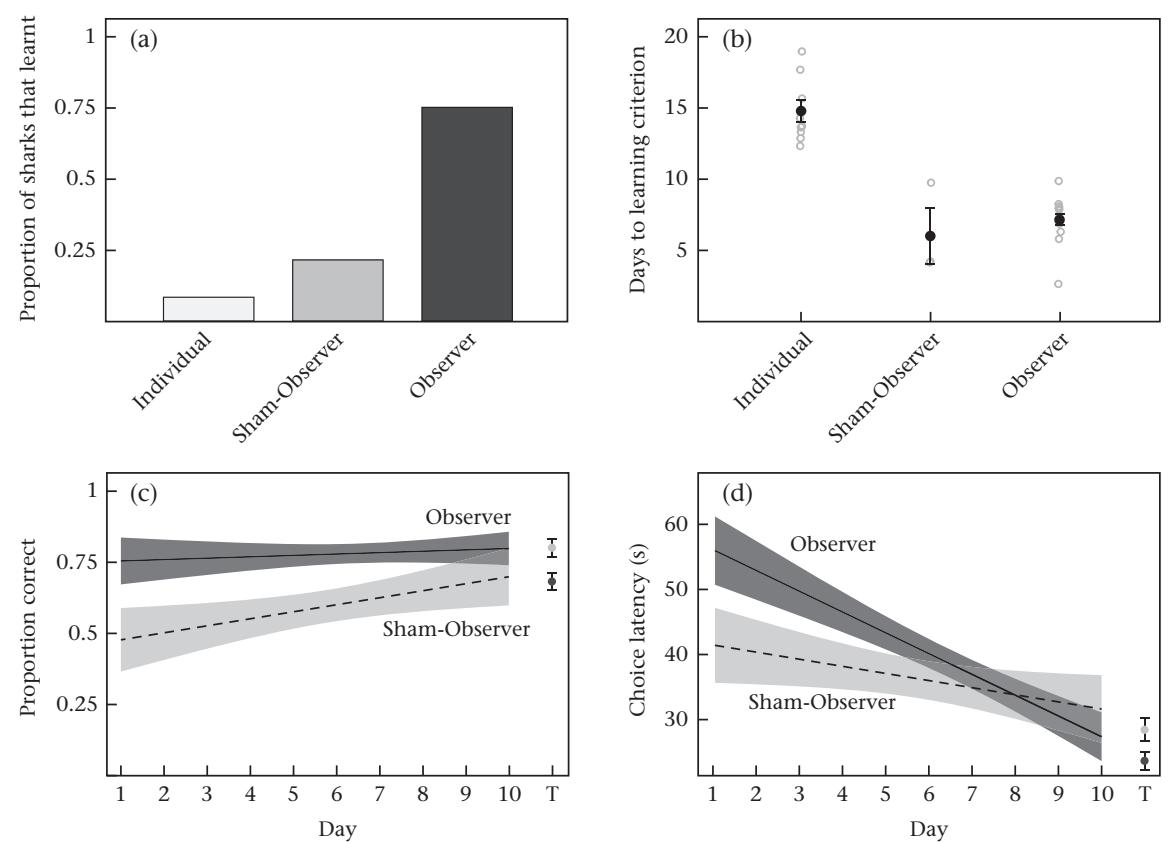

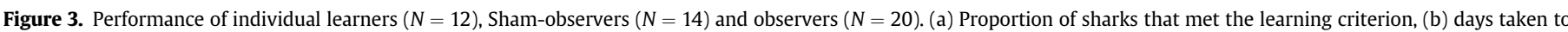
reach criterion, (c) proportion of correct crossings across days and (d) latency of choice across days. $\mathrm{T}=$ test days.

resources, providing some exposure to social cues. Juvenile Port Jackson sharks do not actively group and are seen in the wild solitarily or in dyads, with occasional small, loose aggregations in sheltered locations (Powter \& Gladstone, 2009; Vila Pouca \& Brown, 2019). Therefore, these juveniles may exploit social information in addition to other cues to increase their chances of survival.

Our protocol did not allow us to identify the mechanisms involved in socially learning the task. The results may be explained through social facilitation, local/stimulus enhancement or observational learning (Hoppitt \& Laland, 2008). Observations during the trials suggested that demonstrators were drawing attention to the route and thus increased the observer's learning opportunities; in fewer instances, observers followed the demonstrator directly along the route. Observers and Sham-Observers took the same number of days to learn the task; however, this result may be due to lack of power since only three Sham-Observers reached criterion. To our knowledge, only two other studies have investigated social 
Table 1

Outcomes of statistical models comparing learning treatments

\begin{tabular}{|c|c|c|c|c|}
\hline & $\beta$ & SE & $z$ & $P$ \\
\hline \multicolumn{5}{|l|}{ Number of sharks that learnt $\left(N_{\text {obs }}=46\right)$} \\
\hline Intercept (Observer - 3T) & 1.588 & 0.715 & 2.223 & 0.026 \\
\hline Treatment (Sham-Observer) & -2.502 & 0.866 & -2.891 & 0.004 \\
\hline Treatment (Individual) & -3.625 & 1.198 & -3.027 & 0.002 \\
\hline Training Frequency (6T) & -0.882 & 0.792 & -1.113 & 0.266 \\
\hline \multicolumn{5}{|l|}{ Days to learning criterion $\left(N_{\mathrm{obs}}=27\right)$} \\
\hline Intercept (Observer - 3T) & 2.0359 & 0.1068 & 19.064 & $<0.001$ \\
\hline Treatment (Sham-Observer) & -0.1852 & 0.2549 & -0.727 & 0.467 \\
\hline Treatment (Individual) & 0.7574 & 0.1313 & 5.768 & $<0.001$ \\
\hline Training Frequency $(6 \mathrm{~T})$ & -0.1883 & 0.1275 & -1.476 & 0.140 \\
\hline \multicolumn{5}{|l|}{ Choice of route $\left(N_{\text {ind }}=34 ; N_{\mathrm{obs}}=843\right)$} \\
\hline Intercept (Observer - 3T) & 1.00431 & 0.311 & 3.230 & 0.001 \\
\hline Treatment (Sham-Observer) & -1.0958 & 0.310 & -3.533 & $<0.001$ \\
\hline Day & 0.0776 & 0.031 & 2.507 & 0.012 \\
\hline Training Frequency $(6 \mathrm{~T})$ & -0.135 & 0.299 & -0.452 & 0.651 \\
\hline \multicolumn{5}{|l|}{ Latency of choice $\left(N_{\text {ind }}=34 ; N_{\mathrm{obs}}=843\right)$} \\
\hline Intercept (Observer - 3T) & 7.946 & 0.371 & 21.401 & $<0.001$ \\
\hline Treatment (Sham-Observer) & -1.620 & 0.505 & -3.206 & 0.003 \\
\hline Day & -0.304 & 0.033 & -9.143 & $<0.001$ \\
\hline Training Frequency $(6 \mathrm{~T})$ & -0.058 & 0.376 & -0.154 & 0.879 \\
\hline Treatment ${ }^{*}$ Day & 0.218 & 0.053 & 4.112 & $<0.001$ \\
\hline \multicolumn{5}{|l|}{ Latency of correct choice $\left(N_{\text {ind }}=32 ; N_{\mathrm{obs}}=606\right)$} \\
\hline Intercept (Observer - 3T) & 8.013 & 0.391 & 20.519 & $<0.001$ \\
\hline Treatment (Sham-Observer) & -2.466 & 0.607 & -4.062 & $<0.001$ \\
\hline Day & -0.303 & 0.039 & -7.823 & $<0.001$ \\
\hline Training Frequency (6T) & 0.073 & 0.379 & 0.194 & 0.847 \\
\hline Treatment * Day & 0.284 & 0.071 & 4.018 & $<0.0001$ \\
\hline \multicolumn{5}{|l|}{ Choice of route during test $\left(N_{\text {ind }}=30 ; N_{\text {obs }}=238\right)$} \\
\hline Intercept (Observer - 3T) & 0.776 & 0.319 & 2.433 & 0.015 \\
\hline Treatment (Sham-Observer) & 0.619 & 0.439 & 1.411 & 0.158 \\
\hline Training Frequency (6T) & 0.051 & 0.400 & 0.127 & 0.899 \\
\hline \multicolumn{5}{|l|}{ Null trials over total trials $\left(N_{\mathrm{obs}}=46\right)$} \\
\hline Intercept (Observer - 3T) & -1.046 & 0.721 & -1.451 & 0.147 \\
\hline Treatment (Sham-Observer) & 0.926 & 1.046 & 0.885 & 0.376 \\
\hline Treatment (Individual) & -0.448 & 1.278 & -0.351 & 0.726 \\
\hline Training Frequency $(6 \mathrm{~T})$ & 0.748 & 0.964 & 0.776 & 0.438 \\
\hline Treatment ${ }^{*}$ Training Frequency (Sham-Observer ${ }^{*} 6 \mathrm{~T}$ ) & -0.222 & 1.448 & -0.153 & 0.878 \\
\hline Treatment ${ }^{*}$ Training Frequency (Individual ${ }^{*} 6 \mathrm{~T}$ ) & -1.262 & 1.908 & -0.662 & 0.508 \\
\hline
\end{tabular}

$N_{\text {ind }}=$ number of individuals; $N_{\text {obs }}=$ number of observations; $3 \mathrm{~T}=3$ trials/day; $6 \mathrm{~T}=6$ trials/day. Significant values are given in italics.

learning in elasmobranchs. Juvenile lemon sharks learnt to approach an artificial target by observing and/or interacting over 20 trials with sharks taught to push their snout against the target for a food reward, while their sham-observer counterparts failed to engage with the target (Guttridge et al., 2013). In another study, freshwater stingrays that observed trained conspecifics extracting a food reward from an apparatus performed the task faster (10-30 trials) than individuals that had not observed the task (47-61 trials; Thonhauser et al., 2013). Together with these two studies, our work provides evidence that elasmobranchs can socially learn across a range of contexts (e.g. associative learning, tool use, two-choice discrimination) on a timescale comparable to that for other vertebrates. Considering, as well, the increasing research on individual learning abilities of sharks, it becomes ever more apparent that the cognitive abilities of elasmobranchs are as well developed as those in teleosts and other vertebrates (for a review, see Schluessel, 2015). For example, laboratory studies have shown that, among other abilities, sharks can learn and habituate, have spatial memory and quantitative abilities, can categorize visual objects and recognize different motion patterns (Guttridge \& Brown, 2014; Schluessel \& Bleckmann, 2012; Schluessel \& Duengen, 2015; Schluessel, Kortekamp, Cortes, Klein, \& Bleckmann, 2015; Vila Pouca \& Brown, 2018; Vila Pouca, Gervais, Reed, Michard, \& Brown, 2019). Studies in the wild are scarce; however, the 'goal-directed' navigation of tiger sharks, Galeocerdo cuvier, and thresher sharks, Alopias vulpinus, indicates they may use cognitive spatial maps to move between foraging areas (Papastamatiou et al., 2011), and the decrease in catchability of individual blacktip reef sharks, Carcharhinus melanopterus, suggests they learnt to avoid capture (Mourier, Brown, \& Planes, 2017b).

Contrary to expectations, training frequency did not influence learning performance. This result might be linked to a 'distributedpractice effect'. Distributed practice is a schedule that spreads out study activities over time and is known to improve student success compared to massed practice (Delaney, Verkoeijen, \& Spirgel, 2010; Dunlosky, Rawson, Marsh, Nathan, \& Willingham, 2013). While this phenomenon is complex and relates mostly to memory consolidation (Dunlosky et al., 2013), it suggests that 'more is not always better' when it comes to training animals for cognitive tasks. Most of the learning might occur between sessions; thus, having more trials per day does not necessarily improve learning. For example, higher increases in accuracy between training sessions (compared to within sessions) have been observed in learning tasks with pigeons, Columba livia (I. Fortes \& T. Zentall, personal communication, April 2018). Our small number of trials per day, constrained by the slow metabolism of the study species, did not allow us to detect when the increase in accuracy occurred.

We found high individual variation in task motivation, with an indication that Individual Learners participated more often than Sham-Observers and Observers. This variation could be due to social interactions and/or hierarchical structure between pairs, as social learning seems to depend on factors such as intragroup 
competition and scrounging (Vilhunen et al., 2005). It is also possible that individual personality traits contributed to the variation we observed, as personality (exploration, boldness, activity, aggression and sociability) can affect learning ability across species (Dougherty \& Guillette, 2018). Further work is needed to examine the relationship between personality traits and variation in learning ability in elasmobranchs. We also found that only six Observers maintained performance without demonstration. Of the 14 that failed, eight showed high response rates and low choice latency, indicating they learnt the overall task requirement (cross the partition to obtain food) but failed to retain the correct route. This could have resulted from animals conforming to social conventions in the presence of demonstrators but being released from social constraints in their absence (Brown \& Laland, 2002).

Vertical transmission of information through social learning can lead to cross-generational changes in behaviour that result in unique population culture (Fernö, Huse, Jakobsen, Kristiansen, \& Nilsson, 2011; Rendell et al., 2011). Many shark species, including Port Jackson sharks, show migratory and philopatric behaviour (Bass et al., 2016; Chapman, Feldheim, Papastamatiou, \& Hueter, 2015), and social learning has been suggested as one mechanism that maintains their migration routes (O'Gower, 1995). Further knowledge of what drives elasmobranch aggregations and social group formation and what role social learning and social information diffusion plays in their ecology and behaviour will prove an important avenue for future research.

\section{Author Contributions}

All authors contributed to the study design. C.V.P. and D.H. collected the data. C.V.P analysed the data and drafted the manuscript. All authors revised the manuscript, gave final approval and are accountable for accuracy.

\section{Conflicts of Interest}

We declare we have no competing interests.

\section{Data Availability}

Data and R code are available at Figshare: https://doi.org/10. 6084/m9.figshare.8330627.

\section{Acknowledgments}

We thank Inês Fortes and Thaís Ribeiro for fruitful discussions on the 'distributed-practice effect', Joshua Reed and Alyssa Luongo for help running trials, and Stephanie Bagala and SIMS staff for husbandry assistance. Research funded by Macquarie University, Endeavour Postgraduate Awards (scholarship to C.V.P.), and PADI Foundation (research grant to C.V.P.).

\section{References}

Bass, N. C., Mourier, J., Knott, N. A., Day, J., Guttridge, T., \& Brown, C. (2016). Longterm migration patterns and bisexual philopatry in a benthic shark species. Marine and Freshwater Research, 68(8), 1414-1421.

Brown, C. (2001). Familiarity with the test environment improves escape responses in the crimson spotted rainbowfish, Melanotaenia duboulayi. Animal Cognition, 4(2), 109-113. https://doi.org/10.1007/s100710100105.

Brown, C., \& Laland, K. N. (2002). Social learning of a novel avoidance task in the guppy: Conformity and social release. Animal Behaviour, 64(1), 41-47.

Brown, C., \& Warburton, K. (1999). Social mechanisms enhance escape responses in shoals of rainbowfish, Melanotaenia duboulayi. Environmental Biology of Fishes, 56(4), 455-459.
Chapman, D. D., Feldheim, K. A., Papastamatiou, Y. P., \& Hueter, R. E. (2015). There and back again: A review of residency and return migrations in sharks, with implications for population structure and management. Annual Review of Marine Science, 7(1), 547-570. https://doi.org/10.1146/annurev-marine-010814015730.

Coolen, I., Dangles, O., \& Casas, J. (2005). Social learning in noncolonial insects? Current Biology, 15(21), 1931-1935.

Delaney, P. F., Verkoeijen, P. P., \& Spirgel, A. (2010). Spacing and testing effects: A deeply critical, lengthy, and at times discursive review of the literature. In B. H. Ross (Ed.), The psychology of learning and motivation (Vol. 53, pp. 63-147). San Diego, CA: Elsevier Academic Press.

Dougherty, L. R., \& Guillette, L. M. (2018). Linking personality and cognition: A meta-analysis. Philosophical Transactions of the Royal Society B: Biological Sciences, 373(1756), 20170282.

Dunlosky, J., Rawson, K. A., Marsh, E. J., Nathan, M. J., \& Willingham, D. T. (2013). Improving students' learning with effective learning techniques: Promising directions from cognitive and educational psychology. Psychological Science in the Public Interest, 14(1), 4-58.

Fernö, A., Huse, G., Jakobsen, P. J., Kristiansen, T. S., \& Nilsson, J. (2011). Fish behaviour, learning, aquaculture and fisheries. In C. Brown, K. Laland, \& J. Krause (Eds.), Fish cognition and behavior (2nd ed., pp. 359-404). Oxford, U.K.: Wiley.

Friard, O., \& Gamba, M. (2016). BORIS: A free, versatile open-source event-logging software for video/audio coding and live observations. Methods in Ecology and Evolution, 7(11), 1325-1330. https://doi.org/10.1111/2041-210X.12584.

Galef, B. G., \& Laland, K. N. (2005). Social learning in animals: Empirical studies and theoretical models. AIBS Bulletin, 55(6), 489-499.

Giraldeau, L., \& Lefebvre, L. (1996). Is social learning an adaptive specialization? In C. M. Heyes, \& B. G. Galef, Jr. (Eds.), Social learning in animals: the roots of culture (pp. 107-128). San Diego, CA: Academic Press.

Guttridge, T., \& Brown, C. (2014). Learning and memory in the Port Jackson shark Heterodontus portusjacksoni. Animal Cognition, 17(2), 415-425. https://doi.org/ 10.1007/s10071-013-0673-4.

Guttridge, T., van Dijk, S., Stamhuis, E. J., Krause, J., Gruber, S. H., \& Brown, C. (2013). Social learning in juvenile lemon sharks, Negaprion brevirostris. Animal Cognition, 16(1), 55-64.

Heinrich, D., Vila Pouca, C., Brown, C., \& Huveneers, C. (2019). Effects of reward magnitude and training frequency on the learning rates and memory retention of the Port Jackson shark. Submitted manuscript.

Heyes, C. (2012). What's social about social learning? Journal of Comparative Psychology, 126(2), 193.

Hoppitt, W., \& Laland, K. N. (2008). Social processes influencing learning in animals: A review of the evidence. Advances in the Study of Behavior, 38, 105-165.

Jacoby, D. M., Croft, D. P., \& Sims, D. W. (2012). Social behaviour in sharks and rays: Analysis, patterns and implications for conservation. Fish and Fisheries, 13(4), 399-417.

Kis, A., Huber, L., \& Wilkinson, A. (2015). Social learning by imitation in a reptile (Pogona vitticeps). Animal Cognition, 18(1), 325-331.

Laland, K. N. (2004). Social learning strategies. Animal Learning \& Behavior, 32(1), 4-14.

Lefebvre, L., \& Giraldeau, L.-A. (1994). Cultural transmission in pigeons is affected by the number of tutors and bystanders present. Animal Behaviour, 47(2), $331-337$.

Mourier, J., Bass, N. C., Guttridge, T. L., Day, J., \& Brown, C. (2017a). Does detection range matter for inferring social networks in a benthic shark using acoustic telemetry? Royal Society Open Science, 4(9), 170485.

Mourier, J., Brown, C., \& Planes, S. (2017b). Learning and robustness to catch-andrelease fishing in a shark social network. Biology Letters, 13(3), 20160824. https://doi.org/10.1098/rsbl.2016.0824.

Mourier, J., Vercelloni, J., \& Planes, S. (2012). Evidence of social communities in a spatially structured network of a free-ranging shark species. Animal Behaviour, 83(2), 389-401.

Naylor, G. J. P., Ryburn, J. A., Fedrigo, O., \& López, J. A. (2005). Phylogenetic relationships among the major lineages of modern elasmobranchs. In W. C. Hamlett, \& B. G. M. Jamieson (Eds.), Reproductive biology and phylogeny (Vol. 3, pp. 1-25). Enfield, NH: Science Publishers.

Noble, D. W. A., Byrne, R. W., \& Whiting, M. J. (2014). Age-dependent social learning in a lizard. Biology Letters, 10(7), 20140430. https://doi.org/10.1098/ rsbl.2014.0430.

O'Gower, A. (1995). Speculations on a spatial memory for the Port Jackson shark (Heterodontus portusjacksoni) (Meyer) (Heterodontidae). Marine and Freshwater Research, 46(5), 861-871.

Papastamatiou, Y. P., Cartamil, D. P., Lowe, C. G., Meyer, C. G., Wetherbee, B. M., \& Holland, K. N. (2011). Scales of orientation, directed walks and movement path structure in sharks. Journal of Animal Ecology, 80(4), 864-874. https://doi.org/ 10.1111/j.1365-2656.2011.01815.x.

Powter, D. M., \& Gladstone, W. (2009). Habitat-mediated use of space by juvenile and mating adult Port Jackson sharks, Heterodontus portusjacksoni, in Eastern Australia. Pacific Science, 63(1), 1-15.

R Core Team. (2016). R: A language and environment for statistical computing. Vienna, Austria: R Foundation for Statistical Computing. Retrieved from: https://www. R-project.org/. 
Rendell, L., Fogarty, L., Hoppitt, W. J., Morgan, T. J., Webster, M. M., \& Laland, K. N. (2011). Cognitive culture: Theoretical and empirical insights into social learning strategies. Trends in Cognitive Sciences, 15(2), 68-76.

Schluessel, V. (2015). Who would have thought that 'Jaws' also has brains? Cognitive functions in elasmobranchs. Animal Cognition, 18(1), 19-37.

Schluessel, V., \& Bleckmann, H. (2012). Spatial learning and memory retention in the grey bamboo shark (Chiloscyllium griseum). Zoology, 115(6), 346-353.

Schluessel, V., \& Duengen, D. (2015). Irrespective of size, scales, color or body shape, all fish are just fish: Object categorization in the gray bamboo shark Chiloscyllium griseum. Animal Cognition, 18(2), 497-507.

Schluessel, V., Kortekamp, N., Cortes, J. O., Klein, A., \& Bleckmann, H. (2015) Perception and discrimination of movement and biological motion patterns in fish. Animal Cognition, 18(5), 1077-1091.

Swaney, W., Kendal, J., Capon, H., Brown, C., \& Laland, K. (2001). Familiarity facilitates social learning of foraging behaviour in the guppy. Animal Behaviour, 62(3), 591-598.

Thonhauser, K. E., Gutnick, T., Byrne, R. A., Kral, K., Burghardt, G. M., \& Kuba, M. J. (2013). Social learning in Cartilaginous fish (stingrays Potamotrygon falkneri). Animal Cognition, 16(6), 927-932.

Vila Pouca, C., \& Brown, C. (2018). Food approach conditioning and discrimination learning using sound cues in benthic sharks. Animal Cognition, 21(4), 481-492. https://doi.org/10.1007/s10071-018-1183-1.

Vila Pouca, C., \& Brown, C. (2019). Lack of social preference between unfamiliar and familiar juvenile Port Jackson sharks Heterodontus portusjacksoni. Journal of Fish Biology, 1-7. https://doi.org/10.1111/jfb.13982.

Vila Pouca, C., Gervais, C., Reed, J., Michard, J., \& Brown, C. (2019). Quantity discrimination in Port Jackson sharks incubated under elevated temperatures. Behavioral Ecology and Sociobiology, 73(7), 93. https://doi.org/10.1007/s00265019-2706-8.

Vilhunen, S., Hirvonen, H., \& Laakkonen, M. V. (2005). Less is more: Social learning of predator recognition requires a low demonstrator to observer ratio in Arctic charr (Salvelinus alpinus). Behavioral Ecology and Sociobiology, 57(3), 275-282.

Webster, M. M., \& Laland, K. N. (2017). Social information use and social learning in non-grouping fishes. Behavioral Ecology, 28(6), 1547-1552.

Zuur, A. F., Ieno, E. N., \& Elphick, C. S. (2010). A protocol for data exploration to avoid common statistical problems. Methods in Ecology and Evolution, 1(1), 3-14. https://doi.org/10.1111/j.2041-210X.2009.00001.x.

\section{Appendix}

Before all statistical analyses, we used the protocol from Zuur, Ieno, and Elphick (2010) to explore our data and ensure it fitted model assumptions.

To avoid overfitting, we chose not to include sex and weight in the models. These are not expected to be relevant since our sharks were juveniles (several years away from reaching sexual maturity) and due to our balanced design. We explored the potential effect of housing tank and correct route side (left or right) but did not include them in the final models since they were not significant.

Following the order of the models described above: for models 1-4 we explored an interaction between Training Day and Demonstrator treatment (Demonstrator or Sham-Demonstrator) but chose to exclude them because they were not significant; for model 3 we chose to run a GLMM with Poisson distribution because it had a better fit compared with a zero-inflated Poisson distribution. The response variable for model 2 was log transformed and for models 9 and 10 was square root transformed to improve model fit. We investigated potential interaction effects of Learning Treatment ${ }^{*}$ Training Day and Learning Treatment ${ }^{*}$ Training Frequency but removed them from the final model when they were not significant. The interaction term Learning Treatment ${ }^{*}$ Training Day was included in models 9 and 10 and Learning Treatment ${ }^{*}$ Training Frequency in model 11.
Table A1

Outcomes of statistical models comparing demonstrators and Sham-demonstrators

\begin{tabular}{|c|c|c|c|c|}
\hline & $\beta$ & SE & $z$ & $P$ \\
\hline \multicolumn{5}{|c|}{ First crossing choice $\left(N_{\text {ind }}=22 ; N_{\text {obs }}=994\right)$} \\
\hline Intercept (Demonstrator) & 0.974 & 0.350 & 2.782 & 0.005 \\
\hline Treatment (Sham-Demonstrator) & -1.278 & 0.442 & -2.892 & 0.004 \\
\hline Day & 0.057 & 0.027 & 2.114 & 0.035 \\
\hline \multicolumn{5}{|c|}{ Latency of first choice $\left(N_{\text {ind }}=22 ; N_{\text {obs }}=994\right)$} \\
\hline Intercept (Demonstrator) & 2.997 & 0.128 & 23.348 & $<0.001$ \\
\hline Treatment (Sham-Demonstrator) & 0.562 & 0.164 & 3.424 & 0.003 \\
\hline Day & -0.045 & 0.008 & -5.976 & $<0.001$ \\
\hline \multicolumn{5}{|c|}{ Total partition crossings per trial $\left(N_{\text {ind }}=22 ; N_{\text {obs }}=1527\right)$} \\
\hline Intercept (Demonstrator) & -0.008 & 0.309 & -0.025 & 0.980 \\
\hline Treatment (Sham-Demonstrator) & -1.389 & 0.393 & -3.533 & $<0.001$ \\
\hline Day & 0.009 & 0.009 & 0.945 & 0.345 \\
\hline \multicolumn{5}{|c|}{ Proportion of correct crossings per trial $\left(N_{\text {ind }}=22 ; N_{\text {obs }}=1527\right)$} \\
\hline Intercept (Demonstrator) & 0.080 & 0.439 & 0.182 & 0.856 \\
\hline Treatment (Sham-Demonstrator) & -2.576 & 0.551 & -4.678 & $<0.001$ \\
\hline Day & 0.089 & 0.022 & 3.999 & $<0.001$ \\
\hline
\end{tabular}

$N_{\text {ind }}=$ number of individuals; $N_{\text {obs }}=$ number of observations. Significant values are given in italics.

Table A2

Pairwise comparisons for a significant effect of treatment on the number of sharks that reached learning criterion

\begin{tabular}{lllll}
\hline Contrast & $\beta$ & SE & $z$ & $P$ \\
\hline Sham-Observers - Observers & -2.502 & 0.866 & -2.891 & 0.011 \\
Individual - Observers & -3.625 & 1.198 & -3.027 & 0.007 \\
Individual - Sham-Observers & -1.123 & 1.243 & -0.903 & 0.634 \\
\hline
\end{tabular}

Significant values are given in italics.

Table A3

Pairwise comparisons for a significant effect of treatment on the number of days to reach learning criterion

\begin{tabular}{lllll}
\hline Contrast & $\beta$ & SE & $z$ & $P$ \\
\hline Sham-Observers - Observers & -0.185 & 0.255 & -0.727 & 0.739 \\
Individual - Observers & 0.757 & 0.131 & 5.768 & $<0.001$ \\
Individual - Sham-Observers & 0.943 & 0.253 & 3.731 & $<0.001$ \\
\hline
\end{tabular}

Significant values are given in italics.

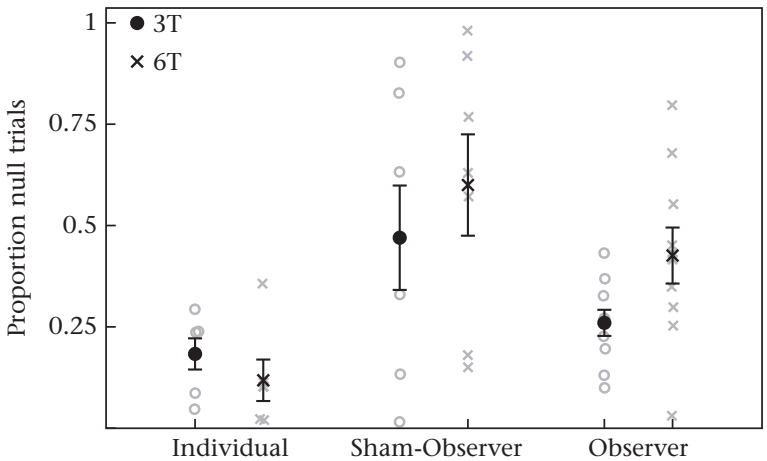

Figure A1. Proportion of trials with no choice (mean \pm SD) per learning treatment (Individual Learners, $N=12$; Sham-Observers, $N=14$; Observers, $N=20$ ) and Training Frequency (3T: 3 trials/day; 6T: 6 trials/day). Individual data are given in grey. 